\title{
Long paths and cycles in faulty hypercubes: existence, optimality, complexity
}

\author{
Tomáš Dvořák, Jiří Fink, Petr Gregor ${ }^{1}$, Václav Koubek ${ }^{2}$ \\ Faculty of Mathematics and Physics, Charles University \\ Malostranské nám. 25, 11800 Prague, Czech Republic
}

\begin{abstract}
A fault-free cycle in the $n$-dimensional hypercube $Q_{n}$ with $f$ faulty vertices is long if it has length at least $2^{n}-2 f$. If all faulty vertices are from the same bipartite class of $Q_{n}$, such length is the best possible. We prove a conjecture of Castañeda and Gotchev [2] asserting that $f_{n}=\left(\begin{array}{l}n \\ 2\end{array}\right)-2$ where $f_{n}$ is the largest integer such that for every set of at most $f_{n}$ faulty vertices, there exists a long fault-free cycle in $Q_{n}$. Furthermore, we present several results on similar problems of long paths and long routings in faulty hypercubes and their complexity.
\end{abstract}

Keywords: hypercube, faulty vertex, long path, long cycle, NP-hard

\section{Introduction}

The $n$-dimensional hypercube $Q_{n}$ is a (bipartite) graph with all binary vectors of length $n$ as vertices and edges joining every two vertices that differ in exactly one coordinate. The application of hypercubes as interconnection networks inspired research of their fault-tolerant properties. Here we consider a problem

1 Partially supported by the Czech Science Foundation Grant 201/08/P298.

2 Partially supported by Grant 1ET100300517 of Information Society.

3 Email: [dvorak@ksvi, fink@kam,gregor@ktiml, koubek@ktiml] .mff.cuni.cz 
of long fault-free cycles and long fault-free paths between two given vertices of a hypercube in which some vertices are faulty.

Let $F$ be a set of faulty vertices of $Q_{n}$. A cycle $C$ of $Q_{n}$ is a long faultfree cycle if it does not contain any faulty vertex and the length of $C$ is at least $2^{n}-2|F|$. A path $P$ of $Q_{n}$ between vertices $u$ and $v$ is a long fault-free path if it does not contain any faulty vertex and the length of $P$ is at least $2^{n}-2|F|-2$. These concepts are motivated by the observation that if all faulty vertices belong to the same bipartite class of $Q_{n}$, then every long fault-free cycle and long fault-free path are the longest possible.

$\mathrm{Fu}$ [6] proved that there exists a long fault-free cycle if $|F| \leq 2 n-4$. Castañeda and Gotchev [2] improved the bound to $|F| \leq 3 n-7$ for $n \geq 5$. The similar problem for paths was first studied by $\mathrm{Fu}$ [7] who showed that there is a long fault-free path in $Q_{n}$ between every two fault-free vertices if $|F| \leq n-2$. Recently, the bound of Fu was improved by Kueng, Liang, Hsu, and Tan [9] to $|F| \leq 2 n-5$, but with an additional (strong) condition that every vertex has at least two fault-free neighbors.

\section{Long paths}

A vertex $u$ is surrounded by $F$ if $F$ contains all neighbors of $u$. Note that if a vertex $u$ is surrounded by $F \cup\{v\}$ or $v$ is surrounded by $F \cup\{u\}$, then there is no long fault-free path of length at least 2 between $u$ and $v$. We proved that this necessary condition is also sufficient if $|F| \leq 2 n-4$.

Theorem 2.1 ([4]) Let $F$ be a set of at most $2 n-4$ faulty vertices of $Q_{n}$ where $n \geq 5$. Then for every two fault-free vertices $u$ and $v$, there exists a long fault-free path between $u$ and $v$ in $Q_{n}$ if and only if $u$ is not surrounded by $F \cup\{v\}$ in $Q_{n}$ and $v$ is not surrounded by $F \cup\{u\}$ in $Q_{n}$.

Moreover, the bound on $|F|$ is tight, as for every $n \geq 5$ there is a configuration of $2 n-3$ faulty vertices and two fault-free vertices $u, v$ satisfying the above necessary condition, but no fault-free path between them is long. Therefore, a natural question arises whether there exists a simple condition on neighbors of end-vertices which allows to significantly increase the upper bound on the number of faulty vertices.

Theorem 2.2 ([3]) Let $F$ be a set of at most $\frac{n^{2}}{10}+\frac{n}{2}+1$ faulty vertices of $Q_{n}$ where $n \geq 15$. Then there is a long fault-free path between every pair of distinct vertices of the largest biconnected component of $Q_{n}-F$.

The bound on $|F|$ is asymptotically optimal in the following sense. Let 
$\psi(n)(\phi(n))$ be the largest integer such that for every set $F$ of at most $\psi(n)$ (resp. $\phi(n))$ faulty vertices of $Q_{n}$ there exists a long fault-free path (resp. between every pair of distinct vertices) in the largest biconnected component of $Q_{n}-F$.

Theorem $2.3([3]) \phi(n) \leq\left(\begin{array}{l}n \\ 2\end{array}\right)-2$ for $n \geq 4$ and $\psi(n) \leq 2\left(\begin{array}{c}n \\ 2\end{array}\right)-1$ for $n \geq 6$.

Putting together Theorems 2.2 and 2.3, we conclude that $\phi(n), \psi(n) \in$ $\Theta\left(n^{2}\right)$. Imposing even more specific condition on end-vertices, we can still increase the upper bound on $|F|$ by a multiplicative constant.

Theorem 2.4 ([5]) If $n \geq 5,|F| \leq \frac{n^{2}+n-4}{4}$, and $u, v$ are two fault-free vertices such that both have at most 3 faulty neighbors, then there exists a long fault-free path between $u$ and $v$.

Furthermore, another such a condition is on the minimal distance in $F$. Clearly, if every two vertices from $F$ are at (Hamming) distance at least 3, then the minimal degree $\delta\left(Q_{n}-F\right)$ in $Q_{n}-F$ is at least $n-1$.

Theorem 2.5 ([8]) Let $F \subseteq V\left(Q_{n}\right)$ where $n \geq 1$ such that $\delta\left(Q_{n}-F\right) \geq n-1$. Then $Q_{n}-F$ contains a long uv-path for every pair of fault-free vertices $u, v$.

\section{$3 \quad$ Long cycles}

Let $f_{n}$ be the largest integer such that for every set of at most $f_{n}$ faulty vertices of $Q_{n}$ there exists a long fault-free cycle. Castañeda and Gotchev [2] noticed, independently on us, that for $n \geq 4$ there is a set $F$ of $\left(\begin{array}{l}n \\ 2\end{array}\right)-1$ faulty vertices such that $Q_{n}$ has no long fault-free cycle. Such a set $F$ may be, for example, formed by all but one vertex at distance 2 from a fixed vertex.

Moreover, they conjectured [2] that $f_{n}=\left(\begin{array}{l}n \\ 2\end{array}\right)-2$. Recently, we proved that the conjecture holds.

Theorem 3.1 ([5]) For every set of at most $\left(\begin{array}{l}n \\ 2\end{array}\right)-2$ faulty vertices, $n \geq 3$, there is a long fault-free cycle in $Q_{n}$.

Furthermore, Theorem 2.5 implies that there is a long cycle in $Q_{n}-F$ if $\delta\left(Q_{n}-F\right) \geq n-1$ and $n \geq 3$.

\section{Routing}

We also extend the problem for more paths that interconnect two given sets $A, B$ of vertices with $A \neq B$ and $|A|=|B|=k$. An $A B$-path is a path 
between a vertex of $A$ and a vertex of $B$. An $A B$-routing is a collection of $k$ vertex-disjoint $A B$-paths. An $A B$-routing $P_{1}, P_{2}, \ldots, P_{k}$ in $Q_{n}-F$ is long if

$$
\left|P_{1}\right|+\left|P_{2}\right|+\cdots+\left|P_{k}\right| \geq 2^{n}-2|F|-k-1 .
$$

Note that for $k=1$, this definition corresponds to long paths. For general $k$, it can be shown that a long $A B$-routing exists in $Q_{n}-F$ for any $F$ only if

$$
b(A \cup B)+b(A \cap B) \leq 2
$$

where $b(C)=|| C \cap X|-| C \cap Y||$ is the balance of $C$, and $X, Y$ are the bipartite classes of $Q_{n}$.

In particular, the (necessary) condition (1) for $k=2$ is equivalent to $b(A \cup B)<|A \cup B|$, i.e. $A \cup B$ is not monopartite. A set $C$ is called monopartite if all vertices in $C$ have the same parity.

Theorem 4.1 ([5]) Let $n \geq 5, F \subseteq V\left(Q_{n}\right), A, B \subseteq V\left(Q_{n}-F\right)$ be such that $|F| \leq\lfloor n / 2\rfloor,|A|=|B|=2, A \neq B$, and $A \cup B$ is not monopartite. Then $Q_{n}-F$ has a long $A B$-routing.

As a consequence, if $F \cup\{u, v\}$ is not monopartite and $|F| \leq\lfloor n / 2\rfloor+1$, we obtain $u v$-paths in $Q_{n}-F$ of length at least $2^{n}-2|F|-1$, which is more than is guaranteed by long paths.

\section{Complexity}

We conclude this survey with a study of computational complexity of our main problems, denoted by $\mathbf{L C}, \mathbf{L P}$ and $\mathbf{L P P}$, and formulated in the following way: Given $n$ and a set $F$ of faulty vertices of $Q_{n}$ (and vertices $u, v$ for the last problem), is there a long fault-free cycle, a long fault-free path, or a long fault-free path between $u$ and $v$, respectively? Considering the applications in data compression [1], for each problem $P \in\{\mathbf{L C}, \mathbf{L P}, \mathbf{L P P}\}$ it is useful to deal also with the variant $P^{\prime}$ where the input is formed by the set of fault-free vertices.

If $|F| \leq\left(\begin{array}{c}n \\ 2\end{array}\right)-2$, the problems $\mathbf{L C}, \mathbf{L} \mathbf{C}^{\prime}, \mathbf{L P}, \mathbf{L P}^{\prime}$ are (trivially) decidable in polynomial time by Theorem 3.1. The next theorem shows that the same conclusion holds even for $\mathbf{L P P}$ and $\mathbf{L P P}^{\prime}$ under a slightly weaker, but still quadratic bound on $|F|$. Moreover, it also shows that the construction of a long fault-free path or cycle takes only constant amortized time per one vertex of the output. 
Theorem 5.1 ([3]) Let $Q_{n}$ contain at most $\frac{n^{2}}{10}+\frac{n}{2}+1$ faulty vertices. There is an algorithm deciding in $O\left(n^{8}\right)$ time whether a long fault-free path $P$ between a given pair of distinct vertices exists. If $P$ exists, the algorithm constructs $P$ in linear time with respect to its length.

On the other hand, in case that the number of faults is not limited, all the problems are intractable.

Theorem 5.2 For every $P \in\{\mathbf{L C}, \mathbf{L P}, \mathbf{L P P}\}, P$ is $N P$-hard and $P^{\prime}$ is NPcomplete.

Moreover, there exists a polynomial $p(n)$ of degree 6 such that $\mathbf{L C}, \mathbf{L P}$, LPP remain NP-hard even if the number of faults is at most $p(n)$.

\section{References}

[1] T. Apaydin, A. S. Tosun, and H. Ferhatosmanoglu. Analysis of basic data reordering techniques. Lect. Notes Comput. Sc., 5069:517-524, 2008.

[2] N. Castañeda and I. S. Gotchev. Embedded paths and cycles in faulty hypercubes. J. Comb. Optim., 2009. doi: 10.1007/s10878-008-9205-6.

[3] T. Dvořák and V. Koubek. Longest paths in hypercubes with a quadratic number of faults. Submitted, 2009.

[4] J. Fink and P. Gregor. Long paths and cycles in faulty hypercubes. Submitted, 2008.

[5] J. Fink and P. Gregor. Long cycles in hypercubes with optimal number of faulty vertices. In preparation, 2009.

[6] J.-S. Fu. Fault-tolerant cycle embedding in the hypercube. Parallel Computing, 29(6):821-832, 2003.

[7] J.-S. Fu. Longest fault-free paths in hypercubes with vertex faults. Information Sciences, 176(7):759-771, 2006.

[8] P. Gregor and R. Škrekovski. Long cycles in hypercubes with distant faulty vertices. Discret. Math. Theor. Comput. Sci., 11(1):185-198, 2009.

[9] T.-L. Kueng, T. Liang, L.-H. Hsu, and J. J. M. Tan. Long paths in hypercubes with conditional node-faults. Information Sciences, 179(5):667-681, 2009. 